Research Article

\title{
Evaluation Algorithm of Teaching Work Quality in Colleges and Universities Based on Deep Denoising Autoencoder Network
}

\author{
Yu Liu \\ Publicity Department (News Center), Xian Peihua University, Xian, China \\ Correspondence should be addressed to Yu Liu; liuyupeihua@126.com
}

Received 10 June 2021; Accepted 19 July 2021; Published 29 July 2021

Academic Editor: Fazlullah Khan

Copyright (c) $2021 \mathrm{Yu}$ Liu. This is an open access article distributed under the Creative Commons Attribution License, which permits unrestricted use, distribution, and reproduction in any medium, provided the original work is properly cited.

\begin{abstract}
One of the most significant components of the teaching department's evaluation of teaching quality is evaluating teachers' performance. With the acceleration of educational informatization, modern information processing technology can be used effectively to evaluate teachers' teaching quality in traditional teaching. In this context, combined with some computational intelligence algorithms, it is critical to developing a targeted teaching quality evaluation system. This paper studies teacher teaching evaluation's characteristics and existing problems and analyzes the fundamental theories and methods of teacher teaching evaluation in colleges and universities. A novel combination of deep denoising autoencoder and support vector machine was proposed for evaluating teacher's teaching quality. Moreover, support vector regression is used to predict the model's output layer to achieve supervised assessment prediction. To capture the data's key properties, the model comprises numerous hidden layers and conducts various feature transformations during unsupervised training to minimize the mean square error between the reconstructed output data and the original input data. As a result, the proposed model achieved the highest recognition accuracy of $85.23 \%$ and convergence compared to other models. Thus, the method can be employed to evaluate and forecast the quality of university teaching activity successfully.
\end{abstract}

\section{Introduction}

The supervision of the classroom teaching process and the quality of classroom teaching [1-3] is an effective strategy for ensuring classroom teaching quality. The mastery and application of knowledge by students is the direct expression of the classroom teaching effect. Therefore, to ensure the quality of classroom teaching, it is necessary to strengthen the control of teaching quality. Teaching evaluation is one of the most commonly used methods in teaching supervision activities [4]. It is an objective inspection and judgment of the teaching process of teachers in class and the learning quality of the students. Through the supervision and assessment of students' learning quality, the implementation results can be fed back to teachers to improve or strengthen certain aspects of teaching and ensure that teachers complete the teaching tasks within the corresponding teaching time limit. To continuously improve students' learning abilities and teachers' teaching abilities, it is necessary to establish a perfect and comprehensive and systematic teaching quality monitoring system [5]. It can help cultivate talents who adapt to the times and have a spirit of innovation and practice.

The establishment of a scientific evaluation system is essential at the moment. Most colleges and universities are still using the traditional mode $[6,7]$ of teaching evaluation. However, the traditional teaching evaluation model has been unable to meet the teaching requirements in the actual teaching process. First, the traditional teaching evaluation model examines the teachers and students only once a semester or at the end of the year. Therefore, students usually have enough time to prepare for the examination. The evaluation results are published at the end of a semester or the beginning of the next semester. The evaluation has no guiding significance for the teaching of this semester. It lacks pertinence for the next semester because the courses and students may have changed in the next semester. Secondly, the evaluation data are intuitive. Traditional evaluation data 
are usually expressed as a set of numbers. Following the students' assessments, some specific data are used to display the students' assessment results.

With the ongoing advancement of information technology and machine learning [1] in today's culture, it is possible to employ machine learning to create a perfect teaching assessment system, which can be used to monitor teachers' teaching in real time. As a result, teachers can know themselves in a shorter time in the teaching process and adjust their teaching plan. Moreover, it can better address the needs of students and teachers in the classroom. The use of machine learning to develop a teaching evaluation system not only is a sign of societal and scientific development but also has the potential to improve teaching quality more rapidly and effectively [2]. This paper uses advanced information and machine learning tools to provide a model for evaluating teachers' teaching quality. The model is based on the combination of deep denoising autoencoder (DAE) and support vector regression (SVR). Then, the model is validated to prove the effectiveness and advantages. The innovations of this article are as follows:

This paper proposed a novel teaching quality evaluation algorithm for colleges and universities based on a deep denoising autoencoder and support vector regression network

The system helps improve teaching quality, promote continuous improvement of teaching goals, and promote scientific decision-making in education

The system can implicitly extract discriminant features of the original data through numerous hidden layers so that the error between the reconstructed output data and the original data is minimized, and the essential properties of the data are obtained

The rest of the paper is organized as follows. Section 2 presents an overall review of the key topics treated and the main works upon which this work is based. Section 3 presents the methodology of the proposed model. Section 4 is about the results, and finally, the conclusion is given in Section 5 .

\section{Background}

The evaluation of teachers can be regarded as a matter within the school. The objective of the education of teachers is to step into society and participate in various social activities. To some extent, the evaluation of teachers is a kind of social behavior. Therefore, the quality of teacher evaluation will directly affect the development process of the future society. In the process of evaluating teachers, the needs of the school's future construction, the needs of social development, and other aspects should be taken into consideration. Today, with the continued popularity of machine learning techniques, a more comprehensive, perfect, and systematic teacher evaluation system can be established with the help of various techniques so that it can make a more objective and referential evaluation of the teaching quality [3].
Teaching quality is essential for a university and college to survive and grow and is a measure tendency toward international higher education. Presently, teaching quality is simply evaluated by weighing the teachers' scores from their students, peers, themselves, and leaders. However, it is critical to determine the real factors that impact teaching quality. With the advent of modern information processing systems, students' evaluation data are accumulated in educational management systems. Traditional methods used to analyze and evaluate these data are usually simple and based on the statistical concept. Also, the deep association analysis is not made to examine several factors impacting the teaching quality evaluation. However, these factors are essential for educational policymakers. It is important to provide decision support for the administrator by effectively investigating and processing these data so that the hidden valued information can be mined.

Machine learning techniques are effective sources to obtain valuable teaching quality information and have been widely applied in various fields, such as telecommunication, insurance, retail sale, and finance. Up to now, many teaching quality evaluation systems based on machine learning techniques have also been developed. Everton et al. [8] developed an adaptive learning system for measuring student outcomes using a deep autoencoder. They pointed out that the proposed adaptive learning system was more effective in providing significant adaptability. However, the system was effective only for evaluating the student's outcomes. Chakrit et al. [9] proposed a voting ensemble method of machine learning to reduce features in the data preprocessing stage and teach sentiment analysis. The results were compared with the existing typical machine learning for sentiment analysis. The experimental results show that the voting ensemble learning fused with chi-square feature selection exhibits higher than typical classifiers. However, they did not apply attribute weights and sentiment analysis to enhance the efficiency of classification. Moraes et al. [10] performed the teaching sentiment classification and compared support vector machine (SVM) against artificial neural network (ANN) algorithms. They discovered the positive and negative sentiments using the learning support vector machines (SVM) and Naïve Bayes (NB) model. The result showed that $\mathrm{ANN}$ provided higher accuracy as compared to SVM and NB. An evaluation system based on the hidden Markov model for evaluating physical education teachers was presented in [11]. The development status of the teaching quality evaluation system of physical education and the applicability of data mining technology and hidden Markov model to evaluating teaching quality in colleges was examined. Finally, a mathematical model for evaluating the quality of physical education teaching in colleges was proposed. Dongjun et al. [12] developed an evaluation prediction model for teachers' teaching quality evaluation based on extreme gradient boosting (XGBoost) algorithm and technology services' ResNet (TS-ResNet). A simulation model was constructed based on the Keras deep learning framework and the results of the proposed model using different convolution kernel sizes, batch sizes, and learning rates were compared. According to the defects existing in the 
traditional evaluation model, this paper will analyze and study it, hoping to find a more reasonable index and set up a more scientific weight for it after various calculations, to establish a more systematic and comprehensive evaluation system.

\section{Methodology}

3.1. Deep Denoising Autoencoder. Deep denoising autoencoder is a common neural network model composed of several noise reduction autoencoders stacked on top of each other, so it has multiple hidden layers [13]. Based on the autoencoder, the denoising autoencoder adds noise to the original input data set, preventing overfitting in the training process and ensuring that the model created after unsupervised training has great robustness and generalization ability. With $x$ as the original input data, $\hat{x}$ as the data after noise reduction, $y$ as the feature vector of the hidden layer data, $z$ as the reconstructed output data, $f$ and $g$ as the encoding and decoding functions, and $L$ as the error function, the schematic diagram of the denoising autoencoder is shown in Figure 1.

In the deep noise reduction autoencoder training process, the input data set is first processed for noise reduction [14]. The general processing method is to set certain input nodes to 0 with a certain probability. Then, using the output of the first noise reduction autoencoder's hidden layer as input to the next hidden layer to realize the data feature conversion between the input layer and the hidden layer, this logic is followed repeatedly using the output of the hidden layer of the previous denoising autoencoder as the input feature vector of the following denoising autoencoder until all the denoising autoencoders have been trained. In this way, after the original data are denoised, the data feature conversion is completed in an unsupervised manner layerby-layer training through all hidden layers. Next, the error between the output layer's reconstructed data and the original data set is calculated using the error function. The backpropagation (BP) algorithm is used to propagate the error to the entire deep noise reduction autoencoder and adjust the option weight and threshold to minimize the error to complete the original input data set. Finally, the original feature vector is recreated. Figure 2 shows the schematic diagram of the deep denoising autoencoder. The Adam algorithm is used to reduce the error between the output data and the original input data in the training process for unsupervised training. Adam is an optimization algorithm for stochastic gradient descent for training in deep learning algorithms. It combines the best properties of the RMSProp and AdaGrad algorithms and can handle sparse gradients on noisy problems.

3.2. Support Vector Machine (SVM). SVM is a statistically based supervised machine learning algorithm. Its goal is to use a classification hyperplane as a decision surface to optimize the isolation edge between positive and negative cases [15-17]. It is mostly used to solve problems such as classification, pattern recognition, and regression. SVM is a

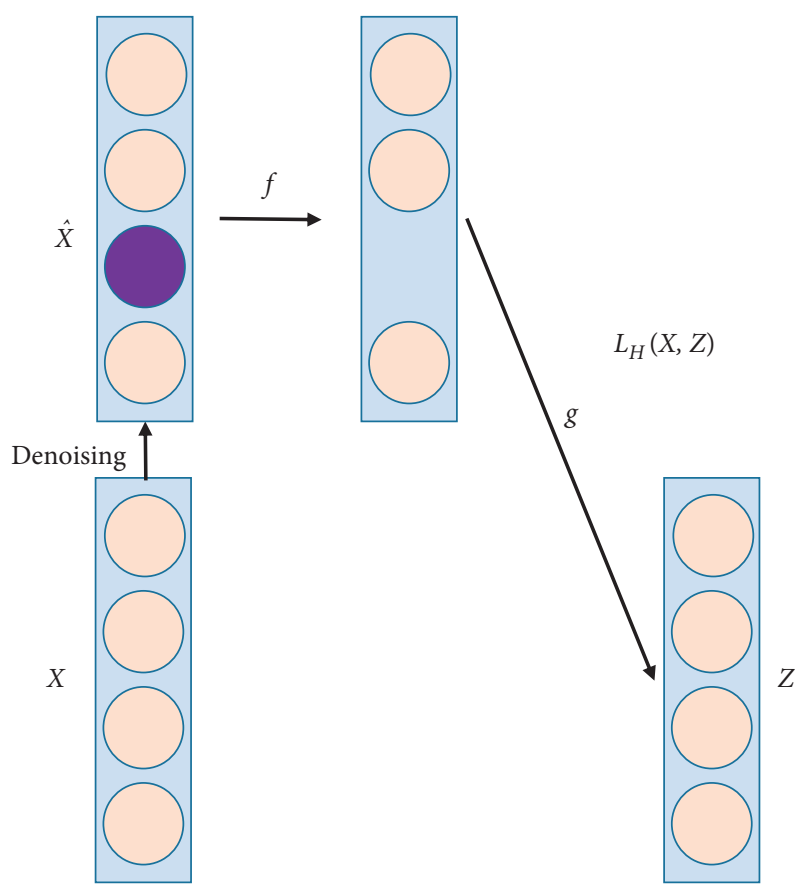

FIgURE 1: Schematic diagram of denoising autoencoder.

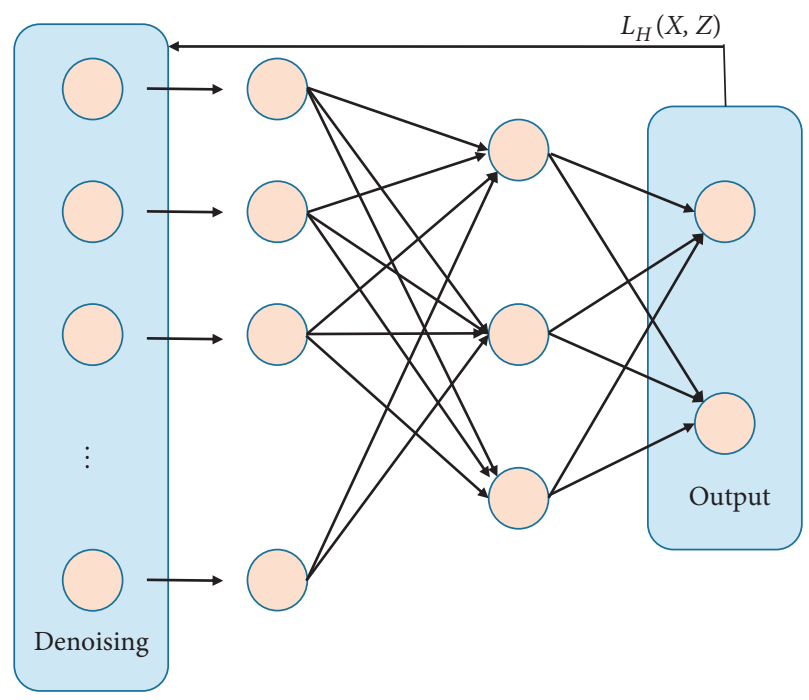

FIgURE 2: Schematic diagram of deep denoising autoencoder.

linear classifier that is used for two-class classification problems. Figure 3 shows the basic architecture of SVM. The idea of SVM classification is based on support vectors. Support vectors are data points that are closed to the hyperplane and affect the position hyperplane. With the help of support vectors, we maximize the margin of the classifier. These are the points that help in building the SVM model. In addressing difficult nonlinear problems and high-dimensional pattern recognition, the support vector machine (SVM) can be reconfigured using kernels.

Support vector regression (SVR) is built based on the concept of SVM [18]. It is one of the general machine learning models that can be used in linearly or nonlinearly separable classification problems. 


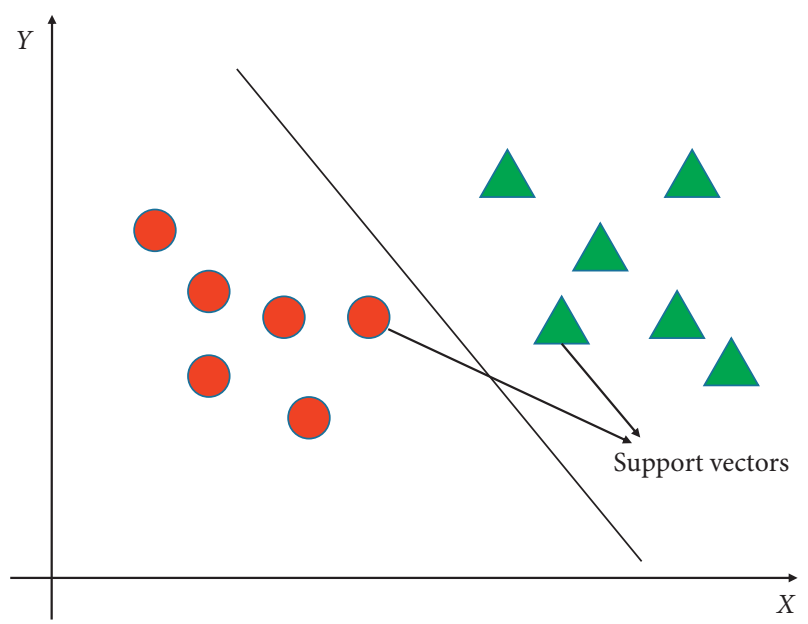

Figure 3: The basic structure of SVM.

Support vector regression is good at fitting nonlinear models and can be used to tackle the teaching evaluation problem [19]. As a result, to evaluate and anticipate the teaching quality evaluation, this study used support vector regression as the predictor of the output layer of the deep neural network model. The goal of support vector regression is to map a complicated nonlinear relationship to a high-dimensional space and then realize a linearized connection in the set high-dimensional space that is similar to the low-dimensional space. Assuming that the dataset is $S=\left\{\left(x_{1}, y_{1}\right),\left(x_{2}, y_{2}\right), \ldots,\left(x_{n}, y_{n}\right) \mid x_{i} \in R^{n}, y_{i} \in R\right\}$, for the data set $s$ that cannot be linearly separated in the original space $R^{n}$, we set a nonlinear mapping function $\varphi$ to map Sto a certain high-dimensional space and make $\varphi(S)$ to have good linear regression characteristics in the feature space $H$. Therefore, for the linearized representation of nonlinear problems, we perform linear regression in the feature space $H$ and then return to the original space $R^{n}$, given a kernel function

$$
K\left(x_{i}, x\right)=\left(\varphi\left(x_{i}\right), \varphi(x)\right) .
$$

The representation of the constructed nonlinear function is

$$
f(x)=\sum_{i=1}^{S}\left(a_{i}-a_{i}^{*}\right) K\left(x_{i}, x\right)+b .
$$

3.3. Deep Denoising Autoencoder and Support Vector Regression. In shallow layer neural network to model addressing the problem of complex computing power and limited capacity, this paper puts forward the depth of the SVM neural network model for solving complex nonlinear problems or dealing with large-scale data set, the depth of the noise reduction automatic coding layer as unsupervised training, and support vector regression (SVM) as a predictive output layer. This proposed model is different from other deep neural networks. The Adam optimization algorithm is applied in the preprocessing of the unsupervised training layer to dynamically adjust the learning step size of each parameter to improve the convergence speed and computing power by using gradient first- and second-moment estimation. [20] Support vector regression is applied in the prediction output layer to achieve a linearization relation in the set high-dimensional space similar to that of the lowdimensional space. The model encoder automatically performs noise reduction on the original input dataset. It applies unsupervised learning step by step training after noise reduction and processing to acquire the original feature vector of the input dataset. In addition, it minimizes the raw input dataset and unsupervised training error between the output data and then uses a support vector regression model for the predictor's final output layer. The input eigenvectors of support vector regression for prediction are the original eigenvectors derived from the dataset.

3.4. DAE-SVM for Teaching Quality Evaluation in Colleges and Universities. The deep structure neural network model contains multiple hidden layers which can obtain the original sample data with excellent computing power. For complex nonlinear problems or to deal with the deep structure of large-scale datasets of neural network models, it is very effective to complete the calculation and modeling [21]. Therefore, the proposed DAE-SVM deep neural network model can be very helpful to solve the teaching quality evaluation in colleges and universities. Suppose we set the input data as $\left(x_{1}, x_{2}, \ldots, x_{n}, y\right)$, where $x$ and $y$ represent feature vector and the labels of target, respectively, and set the total number of samples as $n$. Assume that the weight matrix of each layer of the deep noise reduction autoencoder model is $W$ and the threshold matrix is $b$ and use $\varphi$ to represent the deep noise reduction, autoencoder model. When you input the data set via $\varphi$, then the output feature vector can be computed as

$$
\theta=\varphi(W X+b)
$$

where $\theta$ represents the output feature vector of the deep noise reduction autoencoder model and input $\theta$ into the support vector regression model for evaluation and prediction. Likewise, with $f$, the function of the support vector regression model, the calculation equation is as follows:

$$
y_{d}=f(\theta) \text {. }
$$

The evaluation sample dataset is separated into two parts: a training dataset and a test dataset, with the training dataset being used to train the model. A stable and optimal model is obtained by altering the model's parameters, such as hidden layers, optimization technique, and related support vector regression parameters, to achieve the best prediction efficiency. The test dataset is then entered to ensure that the model is effective in evaluating instructional quality. Figure 4 depicts the flowchart of the DAE-SVM deep neural network model for evaluating teaching quality in colleges and universities.

\section{Experiments and Results}

4.1. Experimental Setup. The hardware platform of this experiment is the Windows 10 system. The processor is Intel(R) Core(TM) i5-6500, and the computer memory is 


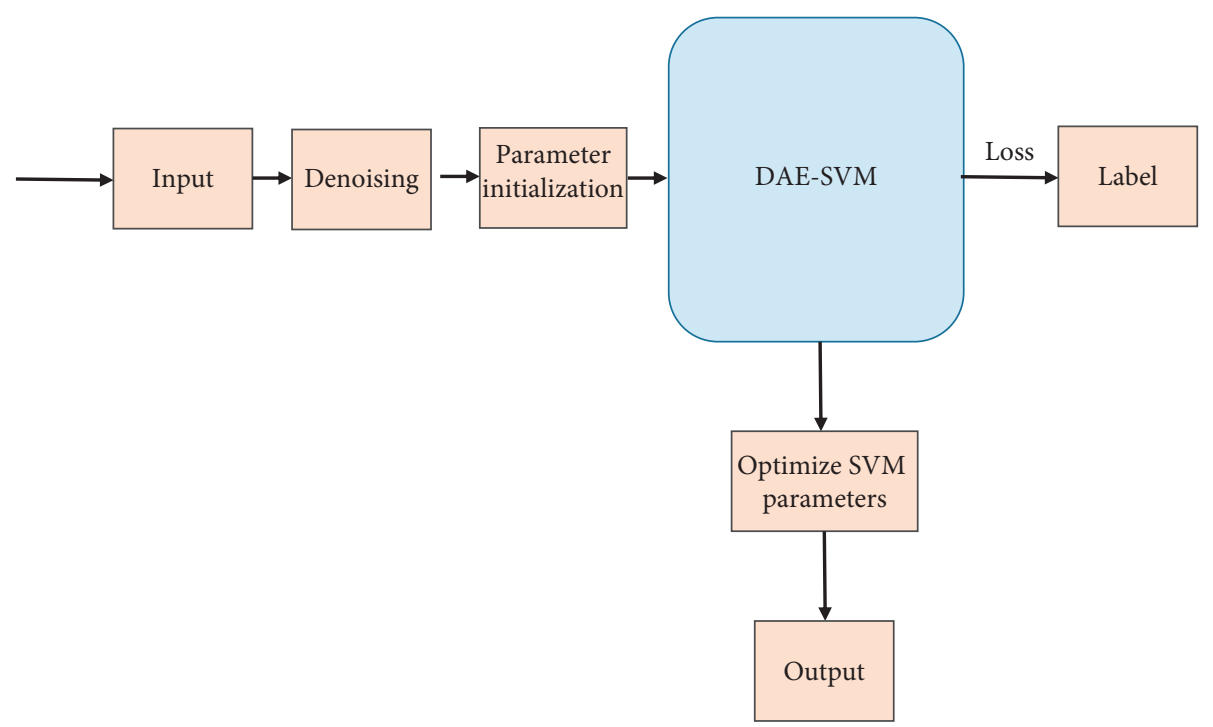

FIGURE 4: Schematic diagram of DAE-SVM deep neural network model for evaluating teaching quality in colleges and universities.

16 GB. The software platform mainly consists of two parts: MATLAB R2014a is used for image preprocessing, and PyCharm builds and trains the network model. The framework for deep learning uses TensorFlow. Using only one TensorFlow API, deep neural network computing may be distributed to any CPUs or GPUs on servers, PCs, or mobile devices.

4.2. Dataset. The evaluation dataset of a course from 2007 to 2020 was obtained from the educational administration system of a university with dataset format $\left(x_{1}, x_{2}, \ldots, x_{n}, y\right)$, with a total of 13,254 samples of data. As the primary body of the evaluation, data from evaluating teachers' instructional processes with students are used as the input value for the assessment model. Because the expected output value of the goal is critical to the model's verification, the comprehensive score is determined by evaluating the teachers' various lectures as the model's expected output value. The sample data with high and low evaluations and inconsistent with the facts were excluded by examining the dataset, and 11,017 samples of data were acquired at the end.

4.3. Evaluation Index. Mean square error (MSE), prediction accuracy, training time, and iteration times (epochs) were used as performance comparison indexes for the proposed DAESVM model and compared with standard BP neural network, decision tree algorithm, support vector machine, and other models. The MSE is the average squared difference between the estimated values and the actual value and is calculated as

$$
\mathrm{MSE}=\frac{1}{n} \sum_{i=1}^{n}\left(Y_{i}-\hat{Y}_{i}\right)^{2},
$$

where $n$ is the number of data points, $Y_{i}$ is the observed value, and $\hat{Y}_{i}$ its predicted value.

Likewise, accuracy is the number of correctly predicted data points out of all the data points and is calculated:

$$
\text { Acc }=\frac{\text { number of correct classified datapoints }}{\text { total number of total datapoints }} \times 100 .
$$

\subsection{Experimental Results}

4.4.1. Accuracy. By learning different deep characteristics, different models produce varied recognition results. To ensure a fair comparison, all experiments were carried out in the same setting and with the same conditions. SVM, Adaptive BP, and DAE were used to compare the suggested model. Table 1 and Figures 5 and 6 illustrate the outcomes of the comparative experiment. SVM reported 8.26 MSE and $78.52 \%$ accuracy, respectively. The lowest MSE was observed in the case of simple DAE with MSE 5.96 and an accuracy of 81.25\%. For Adaptive BP, the MSE and accuracy observed were 7.16 and $80.55 \%$. The lowest MSE and highest accuracy was achieved by the proposed DAE-SVM method with 4.13MSE and $85.23 \%$ accuracy.

4.4.2. Training Time. As the training function of a BP neural network, the improved gradient descent approach is more effective than the classic gradient descent approach and other methods in determining the adaptive learning rate and momentum term. Assuming that the number of neurons in the hidden layer is 20 , the growth ratio and decline ratio of the adaptive learning rate are set as 1.3 and 0.5 , respectively. The momentum term is set as 0.99 . We provided the input dataset into the model for training to obtain a stable network model, then provided a validation test dataset, respectively, in two different algorithms as the training function, and computed the model's performance in terms of MSE and prediction accuracy. Table 2 provides the training time taken by each method. The lowest training time was reported in the case of AdaGrad + Momentum with $36.52 \mathrm{~s}$, whereas the gradient descent method took $56.52 \mathrm{~s}$ in training the model. 
TABle 1: Comparative experimental results of different methods.

\begin{tabular}{lcc}
\hline Method & MSE & Acc (\%) \\
\hline SVM & 8.26 & 78.52 \\
Adaptive BP & 7.16 & 80.55 \\
DAE & 5.96 & 81.25 \\
DAE-SVM & $\mathbf{4 . 1 3}$ & $\mathbf{8 5 . 2 3}$ \\
\hline
\end{tabular}

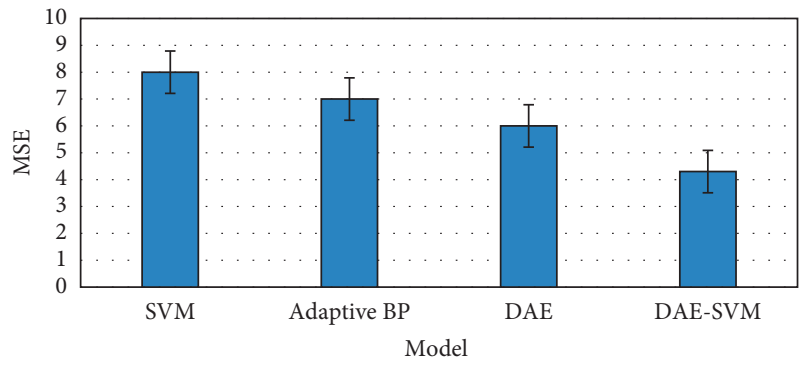

FIGURE 5: Comparative experiment results of different methods on MSE.

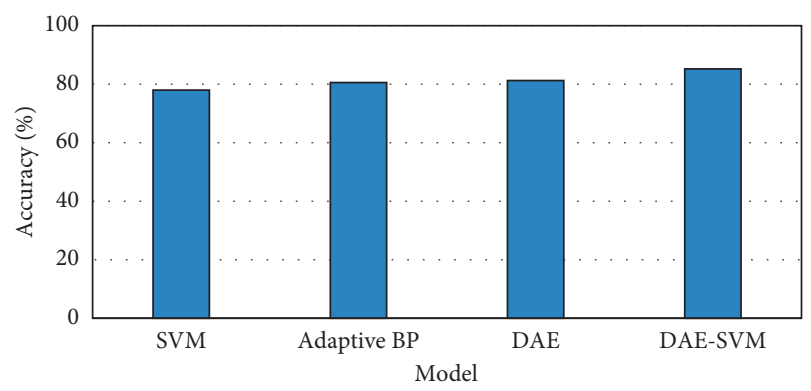

FIGURE 6: Comparative experiment results of different methods on Acc.

TABLE 2: The training time of improved gradient descent.

\begin{tabular}{lc}
\hline Method & Training time \\
\hline Gradient descent & $56.52 \mathrm{~s}$ \\
Adam & $60.35 \mathrm{~s}$ \\
NADAM & $50.34 \mathrm{~s}$ \\
Conjugate gradient & 45.35 \\
RMSPROP & $54.87 \mathrm{~s}$ \\
AdaGrad + momentum & $\mathbf{3 6 . 5 2 ~ s}$ \\
\hline
\end{tabular}

4.4.3. Number of Iteration. An epoch means training the network with all the training data for one complete cycle. In an epoch, we use all of the data exactly once. An epoch comprises one or more batches, where we use a part of the dataset to train the network $[22,23]$. We call passing through the training examples in a batch. In this experiment, the average value is obtained by running the model for 10 iterations. The experimental results are shown in Table 3. The results show that when the adaptive learning rate and the gradient descent method with improved momentum term are used as the training function of the model, the learning rate of the model adaptively and dynamically adjusts according to the change of errors to ensure that the
TABle 3: Performance comparison with different number of epochs.

\begin{tabular}{lccc}
\hline Method & MSE & ACC & Epochs \\
\hline Gradient descent & 11.14 & 77.52 & 10000 \\
Adam & 10.14 & 80.89 & 8000 \\
NADAM & 9.53 & 70.45 & 8500 \\
Conjugate gradient & 8.39 & 60.54 & 7000 \\
RMSPROP & 8.01 & 75.34 & 7300 \\
ADADELTA & 7.24 & 69.34 & 6000 \\
AdaGrad + momentum & 4.13 & 85.23 & 5000 \\
\hline
\end{tabular}

convergence speed of the model can be improved in the training process, and the error between the model and the expected value of the target can be greatly reduced. After 5000 epochs, the accuracy of the proposed adaptive learning approach $[24,25]$ reached up to $85.23 \%$, and the MSE was reduced to 4.13 . The results presented in Table 3 show that it is more effective than the traditional gradient descent method and another method in terms of prediction accuracy and iteration times, which proves the effectiveness of the proposed algorithm.

\section{Conclusion}

One of the most significant components of the teaching department's teaching quality evaluation is teacher teaching evaluation. Using the teaching evaluation auxiliary decisionmaking system in a specific network environment can help to improve the logic and fairness of teaching assessment. In this paper, we studied the characteristics and existing problems of teacher teaching evaluation, analyzed the fundamental theories and methods of university teacher teaching evaluation. We developed an intelligent model for evaluating teachers' teaching quality at colleges and universities. We proposed a novel combination of deep denoising autoencoder and support vector regression model. To achieve the reconstruction between the output data and the original input data, the model was constructed based on numerous hidden layers and performed several feature conversions during the unsupervised training process. To extract the main qualities of the data, the error mean square error was minimized. The model's output layer employs support vector regression as a predictor to achieve supervised assessment and prediction. The proposed model achieved the highest recognition accuracy of $85.23 \%$ as compared to other methods. The proposed model was evaluated to demonstrate the superior performance of the method in improving the decision-making about teaching quality and can effectively assess and forecast the quality of university instruction.

\section{Data Availability}

The data used to support the findings of this study are included within the article.

\section{Conflicts of Interest}

The author declares no conflicts of interest. 


\section{References}

[1] J. Joyce, D. H. Gitomer, and C. J. Iaconangelo, "Classroom assignments as measures of teaching quality," Learning and Instruction, vol. 54, pp. 48-61, 2018.

[2] L. Liang, Q. Yin, and C. Shi, "Exploring proper names online and its application in English teaching in university," ASP Transactions on Computers, vol. 1, no. 1, pp. 24-29, 2021.

[3] I. Nava, J. Park, D. Dockterman et al., "Measuring teaching quality of secondary mathematics and science residents: a classroom observation framework," Journal of Teacher Education, vol. 70, no. 2, pp. 139-154, 2019.

[4] C. Howe, S. Hennessy, N. Mercer, M. Vrikki, and L. Wheatley, "Teacher-student dialogue during classroom teaching: does it impact on student outcomes," Journal of the Learning Sciences, vol. 28, no. 4-5, pp. 462-512, 2019.

[5] W. D. Chawinga, "Taking social media to a university classroom: teaching and learning using twitter and blogs," International Journal of Educational Technology in Higher Education, vol. 14, no. 1, pp. 1-19, 2017.

[6] S. M. Martinelli, F. Chen, A. N. DiLorenzo et al., "Results of a flipped classroom teaching approach in anesthesiology residents," Journal of Graduate Medical Education, vol. 9, no. 4, pp. 485-490, 2017.

[7] R. Kay, T. MacDonald, and M. DiGiuseppe, "A comparison of lecture-based, active, and flipped classroom teaching approaches in higher education," Journal of Computing in Higher Education, vol. 31, no. 3, pp. 449-471, 2019.

[8] E. Gomede, R. M. de Barros, and L. D. S. Mendes, "Deep auto encoders to adaptive E-learning recommender system," Computers and Education: Artificial Intelligence, vol. 2, Article ID 100009, 2021.

[9] C. Pong-Inwong and K. Kaewmak, "Improved sentiment analysis for teaching evaluation using feature selection and voting ensemble learning integration," in Proceedings of the 2nd IEEE International Conference on Computer and Communications, Chengdu, China, May 2016.

[10] R. Moraes, "Document-level sentiment classification: an empirical comparison between SVM and ANN," Expert Systems with Applications, vol. 40, no. 2, pp. 621-633, 2013.

[11] Y. Zeng, "Evaluation of physical education teaching quality in colleges based on the hybrid technology of data mining and hidden Markov model," International Journal of Emerging Technologies in Learning, vol. 15, no. 1, 2020.

[12] G. Dongjun, X. Wang, and J. Liu, "A teaching quality evaluation model for preschool teachers based on deep learning," International Journal of Emerging Technologies, vol. 16, no. 3, 2021.

[13] M. Yu, T. Quan, Q. Peng, Yu Xu, and L. Liu, "A model-based collaborate filtering algorithm based on stacked autoencoder," Neural Computing and Applications, vol. 35, 2021.

[14] Y. Jiang and Y. Wang, "Evaluation of teaching quality of public physical education in colleges based on the fuzzy evaluation theory," Journal of Computational and Theoretical Nanoscience, vol. 13, no. 12, pp. 9848-9851, 2016.

[15] H. Li, "A teaching quality evaluation model based on a wavelet neural network improved by particle swarm optimization," Cybernetics and Information Technologies, vol. 14, no. 3, pp. 110-120, 2014.

[16] X. Yu, D. Zhan, L. Liu, H. Lv, L. Xu, and J. Du, “A privacypreserving cross-domain healthcare wearables recommendation algorithm based on domain-dependent and domainindependent feature fusion," IEEE Journal of Biomedical and Health Informatics, vol. 42, 2021.
[17] C. Dede, "Theoretical perspectives influencing the use of information technology in teaching and learning," in Proceedings of the International Handbook of Information Technology in Primary and Secondary Education, pp. 43-62, Boston, MA, USA, April 2018.

[18] Y. Zhang, W. Li, L. Zhang, X. Ning, and L. Sun, "CNN: adaptive gabor convolutional neural networks with receptive fields for vein biometric recognition," Concurrency and Computation: Practice and Experience, vol. 31, Article ID e5697, 2020.

[19] X. Yu, Y. Chu, F. Jiang, Y. Guo, and D. Gong, "SVMs classification based two-side cross-domain collaborative filtering by inferring intrinsic user and item features," KnowledgeBased Systems, vol. 141, pp. 80-91, 2018.

[20] R. Liu, X. Ning, W. Cai, and G. Li, "Multiscale dense crossattention mechanism with covariance pooling for hyperspectral image scene classification," Mobile Information Systems, vol. 2021, Article ID 9962057, 15 pages, 2021.

[21] Z. Wang, "The evaluation model of teaching quality of modern and contemporary literature in Chinese higher education based on support vector regression algorithm (SVRA)," in Proceedings of the 2018 International Conference on Robots \& Intelligent System (ICRIS), pp. 452-455, IEEE, Changsha, China, May 2018.

[22] X. Ning, X. Wang, S. Xu et al., "A Review of research on cotraining. concurrency and computation: practice and experience," 2021.

[23] Y. Zhang, Y. Chen, X. Bai et al., "Adaptive unimodal cost volume filtering for deep stereo matching," in Proceedings of the AAAI Conference on Artificial Intelligence, pp. 12926-12934, New York, NY, USA, April 2020.

[24] J. Zhang, Y. Liu, H. Liu, and J. Wang, "Learning local-global multiple correlation filters for robust visual tracking with kalman filter redetection," Sensors, vol. 21, no. 4, p. 1129, 2021.

[25] W. Sun, P. Zhang, Z. Wang, and D. Li, "Prediction of cardiovascular diseases based on machine learning," ASP Transactions on Internet of Things, vol. 1, no. 1, pp. 30-35, 2021. 